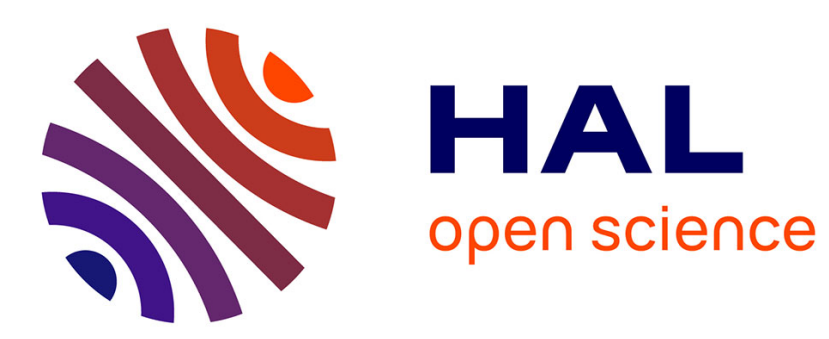

\title{
Adaptive Notch Filters for Prediction of Narrow Band Signals
}

\author{
N. M'Sirdi, Antoine Monneau, Aziz Naamane
}

\section{To cite this version:}

N. M'Sirdi, Antoine Monneau, Aziz Naamane. Adaptive Notch Filters for Prediction of Narrow Band Signals. EFEA 2018 September 24 - 26 in Rome, Italy, Sep 2018, Valencia, Italy. hal-01956284

\section{HAL Id: hal-01956284 \\ https://hal-amu.archives-ouvertes.fr/hal-01956284}

Submitted on 15 Dec 2018

HAL is a multi-disciplinary open access archive for the deposit and dissemination of scientific research documents, whether they are published or not. The documents may come from teaching and research institutions in France or abroad, or from public or private research centers.
L'archive ouverte pluridisciplinaire HAL, est destinée au dépôt et à la diffusion de documents scientifiques de niveau recherche, publiés ou non, émanant des établissements d'enseignement et de recherche français ou étrangers, des laboratoires publics ou privés. 


\title{
Adaptive Notch Filters for Prediction of Narrow Band Signals
}

\author{
Nacer K. M'Sirdi, Antoine Monneau, , and Aziz Naamane,
}

\begin{abstract}
This paper proposes efficient structures of Adaptive Notch Filters (ANF) implemented as constrained ARMA models. These ANF suit very well for fast frequencies tracking and evolutionary spectrum analysis for narrow band and sinusoidal signals in additive noise. Lastly, non-stationary ANF are considered and an estimation procedure implemented.

Index Terms-Adaptive Notch Filters, Narrow band Signals, Frequency Tracking, Spectrum Analysis, Non-stationary Signals, ANC.
\end{abstract}

\section{INTRODUCTION}

\section{A. Objective}

The main interest in this contribution, is the retrieval of the parameters of sinusoidal signal components (amplitudes, phases and frequencies), in noise. We assume (slowly) varying frequencies and varying amplitudes and phases. This is the case when the process is perturbed by the wind (cars, planes, flying robots, wind turbines ...) or the sea waves, swell (boats, submarine robots, wave turbines...). Other examples are the vibrations of processes in mechanics, hydraulics or pneumatics. It is often crucial to detect and predict the vibration signals in order to compensate or tackle their effects.

Recently introduced adaptive control techniques use compensation of the narrow band perturbation signals based on ANF [1], [2], [3], [4]. Autoregressive (AR) and ARMA (Autoregressive with Mean Average) modeling techniques have shown powerful potentialities. They are well suited for recursive signal spectral analysis, interesting for time varying signal spectrum [5], [6], [7].

However, when the noise level is too high and / or when the signal model has poles near or on the unit circle, serious (numerical or stability) problems arise [6].

\section{B. Previous work}

In the narrow-band signals case, successful applications have been realized with AR models [5], [8] but, they need high order autoregressive FIR filter. Very high order AR models has been shown to be asymptotically equivalent to an Adaptive Notch Filter (ANF). Additionally the noise effect is reduced by increasing the order of the AR filter. This adds the expense of computational cost [9]. This has been studied in extensive simulations [8], [10], [7].

Thus, the use of the a priori knowledge of a narrowband feature of the signal, lead us to the design of Notch Filter Structure (NFS) with constrained poles and zeros. The Adaptive Notch Filters (ANF) have been proposed to provide the frequencies estimation and tracking [11] [9], [12]. These estimators can be used by implementation in a cascaded blocks of second order cells.

Some strategies has been proposed to track time varying parameters (like frequencies amplitudes and phases), which may be used in line [13]. $\mathrm{ANF}(\mathrm{s})$ are used to estimated the frequency components in a first step. Then, in a second step, after frequencies tracking the estimated frequencies are used to provide the estimation of amplitudes and phases. This can also be implemented in a recursive manner.

The proposed recursive algorithm consists in two steps. The first step involves a Recursive Maximum Likelihood algorithm to adapt the cascaded filters parameters [7], which will provide the frequencies estimates. The second step uses the last estimates, and then the estimations of amplitudes and phases are given by a Recursive Extended Least Squares (RELS) algorithm .

A theoretical analysis of these algorithms, have been presented in [6], [10] and the applicable estimation methods are reviewed in [10], [11] [14], [9], [15]. These last structures introduce in Parameter Adaptation Algorithms (PAA) some nonlinearity which may give some problems in the transient period of the estimation. The cascade implementation of second order sub models, have been claimed possible.

\section{Contribution of this work}

In this paper clarifies the rationale behind the Adaptive Notch Filters that we have proposed in the past and revisited in this paper. We give a complete theoretical analysis and prove the convergence of the two estimation algorithms:n one to estimate the frequency components by an ARML algorithm and the second to estimate the amplitudes and phases by an RLS algorithm with exponential weighting and a forgetting factor. These algorithms are applied for adaptive prediction of the swell perturbations on a boat.

In section II of this paper we present the Recursive estimation algorithms and the filter structure. We then develop an implementation of the cascaded ANF. The Prediction Error Method (PEM) is applied. This application leads to an Approximate Recursive Maximum Likelihood (ARML) algorithm for frequencies estimation. In a second step, using these estimates, a Recursive Least Squares (RLS) algorithm is employed for the estimation of amplitudes and phases.

The theoretical analysis of these algorithms and their performance evaluation is presented in section III.

Section for will be dedicated to effective Prediction of narrow band signals and parameters estimation. The narrow 
band signals, considered are the sea waves. After the ANF for the signal Prediction, we estimate the frequencies and the deduce the estimations of the amplitudes and phase. The algorithms Validation is done by using the swell signals perturbing a boat motion.

\section{RECURSIVE ESTIMATION ALGORITHMS AND FILTER STRUCTURE}

The proposed recursive estimation procedure consists of two stages, as the parameters to estimated are in non linear combination. The first step processed involves an ANF structure implemented in cascaded form for the estimation of the signal frequencies. An Approximate Recursive Maximum Likelihood (ARML) method is developed and used. The second step uses Recursive Instrumental Variables (RIV) to avoid non linearities and get an appropriate model for linear prediction techniques. This leads us a model linear in its parameters (to be estimated). An adaptive estimation algorithm, based on Weighted Recursive Least Squares (WRLS), is used to provide the amplitudes and phases estimations.

The involved signals may be modeled as follows where $v_{k}$ is the noise disturbance, $C_{i}(k)$ and $\beta_{i}(k)$ are, respectively, the time varying amplitude and phase of the frequency component $f_{i}(k)$. All the parameters are susceptible to vary in time. $T_{e}$ is the sampling period.

$$
y_{k}=\sum_{i=1}^{p} C_{i}(k) \cdot \operatorname{Sin}\left(2 \cdot \pi f_{i} \cdot T_{e} \cdot k+\beta_{i}(k)\right)+v_{k}
$$

\section{A. Amplitudes and Phases Estimation with RIV-WRLS}

In this step, we assume that the frequencies of the signal are known (well estimated by the proposed ARLM algorithm) and we develop an estimator for the amplitudes and phases of each frequency component. We use a Weighted Recursive Least Squares algorithm, as in [11], to estimate the amplitude and phase of each sinusoid. The frequencies estimations are provided by the ANF, as will be developed in the next subsection in a previous step.

The signal Model of equation (1 ), can be decomposed in Fourier Series. It may be written as linear combination of the Fourier basis elements (which are the Instrumental Variables (IV) to be used in estimation):

$$
y_{k}=\sum_{l=1}^{p}\left(g_{l} \operatorname{Cos}\left(2 \pi f_{l} T_{e} \cdot k+h_{l} \operatorname{Sin}\left(2 \pi f_{l} T_{e} \cdot k\right)\right)+v_{k}\right.
$$

where the amplitudes $C_{l}$ and phases $\beta_{l}$ for $(l=1 \ldots p)$ are given from the Fourier series parameters by

$$
C_{l}=\sqrt{g_{l}^{2}+h_{l}^{2}} \quad \text { and } \quad \tan \left(\beta_{l}\right)=g_{l} / h_{l}
$$

It is worthwhile to note that the Fourier series parameters are identifiable. We can then define the parameter $\hat{\theta}(k)$ and the regression (by Recursive Instrumental Variable RIV) $\psi \hat{(k)}$ vectors as follows

$$
\left\{\begin{array}{l}
\hat{\theta}(k)=\left[g_{1}, g_{2}, \ldots, g_{p}, h_{1}, h_{2}, \ldots, h_{p}\right]^{T} \\
\Phi_{k}=[C, S]^{T} \text { with } \\
C=\left[\operatorname{Cos}\left(2 \pi f_{l} \cdot T_{e} \cdot k\right) \ldots, \operatorname{Cos}\left(2 \pi f_{p} \cdot T_{e} \cdot k\right)\right] \\
S=\left[\operatorname{Sin}\left(2 \pi f_{l} \cdot T_{e} \cdot k\right), \ldots, \operatorname{Sin}\left(2 \pi f_{p} \cdot T_{e} \cdot k\right)\right]
\end{array}\right.
$$

The parameters are then estimated using an WRLS algorithm as follows:

$$
\left\{\begin{array}{l}
y_{k}^{0}=\hat{\theta}(k-1)^{T} \cdot \Phi_{k} \\
\varepsilon^{0}(k)=y_{k}-\hat{\theta}(k-1)^{T} \cdot \Phi_{k} \\
G_{k}=\frac{1}{\lambda_{0}}\left(G_{k-1}-\frac{G_{k-1} \Phi_{k} \Phi_{k}^{T} G_{k-1}}{\lambda_{0}+\Phi_{k}^{T} G_{k-1} \Phi_{k}}\right) \\
\hat{\theta}(k)=\hat{\theta}(k-1)+G_{k} \cdot \Phi_{k} \cdot \varepsilon^{0}(k) \\
C_{l}=\sqrt{g_{l}^{2}+h_{l}^{2}} \tan \left(\beta_{l}\right)=g_{l} / h_{l}
\end{array}\right.
$$

where $y_{k}^{0}$ is the a priori prediction and $\epsilon^{0}(k)$ is the a priori prediction error. In equation (5) filtered versions of the signal $y_{k}$ can be used; For example, we can use the prediction (of the ANF) produced by the frequency estimation stage. Doing this we will get the RIV coupled with the signal Output Prediction Error Method (RIV-OPEM).

We can note, also, that the RIV regression vector will verify the Persistent Excitation Condition (PEC). The use of a constant $\lambda_{0}$ as an exponential forgetting factor will help for the alertness of the amplitudes and phases estimation by WRLS. The adaptation gain $G_{k}$ will not decrease to zero and remain positive. Then estimation will remain correct in case of slowly time varying parameters.

\section{B. Frequency estimation whit cascaded ANF}

Adaptive Notch Filters are very well suited for estimation of the sinusoidal component frequencies. The $i^{t h}$ sinusoidal component will be filtered by the $i^{t h}$ second order ANF:

$$
H_{i}\left(z^{-1}\right)=\frac{1+a_{i} z^{-1}+z^{-2}}{1+r \cdot a_{i} z^{-1}+r^{2} z^{-2}}
$$

The last equation presents a second order notch filter. Let us note that the transfer function does not depend on amplitudes and phases. Those informations are in the initial conditions. The notch bandwidth is defined by the parameter $0<\mathrm{r}<1$. The parameter $a_{i}$ depends on the notch frequency

$$
a_{i}=-2 \cos \left(2 \pi f_{i} T_{e}\right)
$$

(for $i=1 \ldots p$ ). As they are $\mathrm{p}$ sinusoidal components in equation (1), let us consider the notch filter transfer function as p cascaded second order Notch cells:

$$
H\left(z^{-1}\right)=\prod_{i=1}^{p} H_{i}\left(z^{-1}\right)
$$

With no loss of generality we assume identical bandwidth parameter $r$ for the notches. Thus independence between frequencies yields independence between the parameters of each second order cell. Then each cell can be adapted independently of the others after pre-filtering the signal by the others. For comb filter, relations between the frequencies can be used 
to reduce the computational burden. If all the frequency components are independent we can write

$$
\widetilde{y}_{k}^{j}=\prod_{\substack{i=1 \\ i \neq j}}^{p} H_{i}\left(q^{-1}\right) y_{k}
$$

If we suppose that the cascaded filters $H_{i}\left(z^{-1}\right)$ with central frequency $f_{i}$ (for $i=l \ldots p$ and $i \neq j$ ) have converged then the filter $H_{j}\left(z^{-1}\right)$ will remove one of the $j^{t h}$ sinusoidal components, remaining after the filters $H_{i}\left(z^{-1}\right)$, for $i=1 \ldots p$ and $i \neq j$.

$$
\widetilde{y}_{k}^{j}=\prod_{\substack{i=1 \\ i \neq j}}^{p} \frac{1+a_{i} q^{-1}+q^{-2}}{1+r . a_{i} q^{-1}+r^{2} q^{-2}} \cdot y_{k}
$$

This means that in the signal $\widetilde{y}_{k}^{j}$ the $\mathrm{p}-1$ sinusoidal components have been removed by the corresponding notches and then the prediction error $\varepsilon_{k}$ is obtained by removing the $j^{\text {th }}$ one:

$$
\varepsilon_{k}=H_{j}\left(q^{-1}\right) \widetilde{y}_{k}^{j}=\frac{1+a_{j} q^{-1}+q^{-2}}{1+r . a_{j} q^{-1}+r^{2} q^{-2}} \widetilde{y}_{k}^{j}
$$

Thus, there will exit a unique global minimum for the Mean Squared Error (MSE) Criterion, as each notch remove one frequency component. Following the approach of the Output Prediction Error Method (OPEM), for the estimation algorithm, we obtain as the estimation gradient:

$$
\psi_{k-1}^{j}=-\frac{d \varepsilon_{k}}{d a_{j}}=\frac{(1-r)\left(1-r q^{-2}\right)}{1+r . a_{j} q^{-1}+r^{2} q^{-2}} \cdot \widetilde{y}_{k-1}^{j}
$$

The RML algorithm may be summarized by the following equations, where $F_{k}^{j}$ is the adaptation gain and $0<\lambda<1$ the forgetting factor:

$$
\left\{\begin{array}{l}
\text { for } j=1, \ldots, p, d o \\
\hat{a}_{k}^{j}=\hat{a}_{k-1}^{j}+F_{k-1}^{j} \cdot \psi_{k-1}^{j} \cdot \varepsilon_{k} \\
F_{k}^{j}=F_{k-1}^{j} /\left(\lambda+\psi_{k-1}^{j} \cdot F_{k-1}^{j} \cdot \psi_{k-1}^{j}\right)
\end{array}\right.
$$

The computational complexity of the RML algorithm comes essentially from the computation of the gradient and the successive filtering (equations 10,11,12). A first approximation can be done to reduce computational complexity. It yields an Approximate RML (ARML) algorithm. We can then use the formula (using equation II-B instead of 12):

$$
\psi_{k-1}^{j} \simeq \frac{s_{k-1}^{j}-r \varepsilon_{k-1}}{1+r \cdot a_{j} q^{-1}+r^{2} q^{-2}} \cdot \widetilde{y}_{k}^{j}
$$

with the signals $s_{k}^{j}$ computed as follows

$$
\left\{\begin{array}{l}
\text { for } j=(p-1), \ldots, 1 d o \\
y_{k}^{j}=\prod_{l=j+1}^{p} H_{l}\left(q^{-1}\right) \cdot y_{k} \\
s_{k}^{j}=y_{k}^{j}-y_{k}^{j-1}
\end{array}\right.
$$

Another suggestion is to adapt the Notch filter second order cells successively for $i=1, . ., p$ in a cascaded way or recur- sively. Each cell get as input the output error computed from the previous cells only, in a recursive manner. The bandwidth or what we call the de-biasing parameter $r_{k}$ must be a positive. In our experience we choose this parameter exponentially time varying, from 0 or $r_{0}$ to one (or to $r_{f}$ ), according to the following expression: $r_{k}=r_{d} \cdot r_{k-1}+\left(1-r_{d}\right) \cdot r_{f}$.

\section{ANALYSis AND PERFormance EVAluAtion}

\section{A. Convergence of the ANF (OPEM RML)}

1) Second order ANF with an RML algorithm: Let us first consider the case of a single cell $H_{j}\left(z^{-1}\right)$ (see (6)) driven by the signal defined in (1) with $p=1$.

Assume, either if $p>1$, that the preceding filters $H_{i}\left(z^{-1}\right)$ (for $i=1 . . j-1, j+1, . . n$ and $i \neq j$ ) have, all of them, converged to their optimal value. The bandwidth, of the filter $H_{i}\left(z^{-1}\right)$ are assumed large enough to remove (or attenuate) the other frequencies $\left(f_{i}\right.$ respectively $i \neq j$ ).

Thus $\tilde{y}_{k}^{j}$ is composed by a single frequency $f_{j}$ plus an additive independent noise $\eta_{k}$.

$$
\tilde{y}_{k}^{j}=C_{j}(k) \cdot \operatorname{Sin}\left(2 . \pi f_{j} \cdot T_{e} \cdot k+\beta_{j}(k)\right)+\eta_{k}
$$

Tacking for the signal an AR model with two complex conjugate poles on the unit circle and applying the RML Parameter Estimation algorithm, the a posteriori error can be written:

$$
\varepsilon_{k}=\frac{\hat{A}_{j}\left(r q^{-1}\right)}{A_{j}\left(r q^{-1}\right)} \cdot\left(\hat{a}_{j}-a_{j}\right) \cdot \psi_{k-1}^{j}+\frac{\eta_{k}}{A_{j}\left(r q^{-1}\right)}
$$

with

$$
\begin{gathered}
\tilde{y}_{k}^{j}=-a_{j} \tilde{y}_{k-1}^{j}-\tilde{y}_{k-2}^{j}+\eta_{k} \\
A_{j}\left(r q^{-1}\right)=1+r \cdot a_{j} q^{-1}+r^{2} q^{-2} \\
\hat{A}_{j}\left(r q^{-1}\right)=1+r . \hat{a}_{j} q^{-1}+r^{2} q^{-2}
\end{gathered}
$$

It is well known, in the general case, that the RML algorithm needs a stability monitoring. For the ANF this procedure can be removed in virtue of the following Lemma. We use a time varying de-biasing parameter.

Lemma 1. Let $r_{k}$ be a positive (bandwidth) parameter exponentially time varying, from 0 to one, according to the following expression:

$$
r_{k}=r_{d} \cdot r_{k-1}+\left(1-r_{d}\right) \cdot r_{f}
$$

Then there exit positive constants, less than $1,\left(r_{0}, r_{d}, r_{f}\right)$ such that $\hat{A}\left(r_{k}, q^{-1}\right)$ is infinitely often stable.

Proof: The bandwidth (or de-biasing) parameter $r_{k}$ acts like a contraction of the poles, attracting them toward the center of the unit circle. In addition $r_{k}$ starts at $r_{0}$, for $\mathrm{k}=0$, and goes to $r_{f}$, when $\mathrm{k}$ goes to infinity. We can then take $r_{f}$ less than the inverse of the maximum modulus of the unstable poles of $\hat{A}\left(r_{k}, q^{-1}\right)$.

Now applying the theoretical background, on convergence of adaptation algorithms, presented in [16] and particularly the Theorem 4 of [16] leads to the following Theorem to establish the convergence of the ANF OPRM RML algorithm. 
Theorem 2. : Under the following assumptions:

- $\hat{A}\left(r_{k}, q^{-1}\right)$ is infinitely often stable $\left(r_{k} . \hat{a}_{j}(k)<2\right)$

- for some $\hat{a}_{j}$ fixed $\psi_{k}^{j}, \varepsilon_{k}$ and $\frac{\eta_{k}}{A_{j}\left(r q^{-1}\right)}$ are quasi stationary signal.

- $\eta_{k}$ is independent of $\psi_{k-1}^{j}$ and

- the transfer function $\frac{\hat{A}\left(r z^{-1}\right)}{A\left(r z^{-1}\right)}-\frac{1}{2}$ is SPR (Strictly Positive Real).

Then we get, for the estimation algorithm, the following:

- $D_{c}=\left\{a\right.$ such as $\left.E\left\{\left(\hat{a}_{j}-a_{j}\right) \cdot \psi_{k-1}^{j}\right\}=0\right\}$ is the convergence domain

- $\lim \left(\epsilon_{k}-\eta_{k}\right)=0$ WP1 (With probability one)

- $\lim \left(\hat{a_{j}}(k)\right) \epsilon D_{c} W P 1$ (With probability one)

To ensure the SPR condition we consider the following lemma.

Lemma 3. : There always exist an $r$ such that $\frac{A\left(r \cdot \hat{q}^{-1}\right)}{A\left(r \cdot q^{-1}\right)}$ is a Strictly Positive Real transfer function (SPR).

The last condition on $r$ is less restrictive than the one for the stability. We can then conclude that the Lemmas 1 and 2, with the last theorem, allow us to assert the global stability of the ANF if $\mathrm{r}$ is appropriately chosen such that $\hat{A}\left(r \cdot q^{-l}\right)$ is always stable during estimation progress.

2) Second order ANF with an ELS algorithm: If the ELS estimation method is applied, the estimation error becomes [13]:

$$
\varepsilon_{k}=\frac{1}{A_{j}\left(r q^{-1}\right)} \cdot\left(\hat{a}_{j}-a_{j}\right) \cdot \psi_{k-1}^{j}+\eta_{k}
$$

There exits an upper limit $l$ for the de-biasing parameter in order to get the SPR condition. This limit $l$ depends on the signal frequency and limits the accuracy of the estimation. The SPR condition on $r$ is more restrictive than the stability one. Then Theorem 2 cannot be applied in all cases.

Thus the local stability cannot be established although the global stability can be ensured.

3) Uniqueness of the RML estimates: In theorem 2 we have got asymptotically WPI: $\varepsilon_{k}=\frac{\eta_{k}}{A_{j}\left(r q^{-1}\right)}$

Equations 4 and 14 yield

$$
\varepsilon_{k}=\frac{\hat{A}_{j}\left(q^{-1}\right)}{\hat{A}_{j}\left(r q^{-1}\right)} \cdot \tilde{y}_{k}^{j}=\frac{\hat{A}_{j}\left(q^{-1}\right)}{\hat{A}_{j}\left(r q^{-1}\right)} \cdot \frac{\eta_{k}}{A_{j}\left(q^{-1}\right)}
$$

It then follows from 17 and 18 that:

$$
\frac{\hat{A}_{j}\left(q^{-1}\right)}{\hat{A}_{j}\left(r q^{-1}\right)}=\frac{A_{j}\left(q^{-1}\right)}{A_{j}\left(r q^{-1}\right)}
$$

and then, $\hat{a_{1}}=a_{1}$ with probability one (WP1) if $r \neq 1$.

Theorem 4. : : If the order is correctly chosen (the number of the frequency components is matched), then the RML estimates for ANF are unique

The main results concerning ANF are then:

1) The global convergence can be ensured with an appropriate choice of $r$ the de-biasing parameter without any stability monitoring.

2) The RML estimates are unique.
For $\mathrm{p}$ cascaded cells adapted along the same procedure, each cell will converge near to a local minimum. The filtering by ANFs will make these minima distinct, removing, for each cell, one of the other frequencies. This will not be the case if the implementation of the Notch cells is made in parallel instead of in cascaded form.

\section{B. Convergence of Amplitudes and phases estimation, RIV-} WRLS

The Exponential Convergence of the estimation of the amplitudes and the phases is guaranteed by the WRLS algorithm properties, as shown in the following theorem which uses the concept of persistent excitation see eg [5], [7].

\section{Theorem 5. Convergence of the WRLS algorithm}

The WRLS estimation scheme of equations (II-A) with an exponential forgetting factor $\lambda$ is exponentially stable, under persistent excitation condition.

Proof. The proof of this theorem can be conducted in the same lines as in [17].

The regression vectors (RIV) are composed by $\mathrm{p}$ sinusoids having different frequencies and phases. Then the Persistent Excitation Condition (PEC) is satisfied.

Equation (23) yields by lemma 1 of [17]. The regressors are composed by p sinusoids having different frequencies. Then the persistent excitation condition is satisfied:

$$
0<\gamma_{1} . I<\sum_{k=j}^{j+s} \Phi_{k} \Phi_{k}^{T}<\gamma_{2} . I<\infty
$$

Equation (23) yields by lemma 1 of [17]:

$$
0<\frac{\gamma_{1} \cdot\left(\lambda^{-1}-1\right)}{\left(\lambda^{-(s+1)}-1\right)} \cdot I<F_{k-1}^{-1}<\frac{\gamma_{2}}{1-\lambda^{s+1}} \cdot I+o\left(\lambda^{k}\right)
$$

The adaptation gain is bounded and will never be zero (the alertness property of WRLS and its tracking capabilities). Finally reasoning as in [17] we obtain:

$$
\left\|\tilde{\theta}_{k}\right\|^{2}<\frac{\lambda^{s+1}-1}{\gamma_{1}\left(\lambda^{-1}-1\right)} \cdot \lambda^{k} \cdot \lambda_{\max }\left(F_{0}^{-1}\right) \cdot\left\|\tilde{\theta}_{0}\right\|^{2}
$$

This means that the parameter estimation error will be always strictly decreasing. Then the convergence of the estimations.

Comparative Simulation results will be presented in the final version of this paper.

\section{Simulations AND ExPERIMENTAL VALIDATION}

In order to test the proposed ARML and RLS for predicting narrow band signals, we consider simulation of non-stationary signal with 3 frequency components and an additive centered and normal white noise $v_{k}$ (with variance $\sigma_{v}^{2}=1$ ) and signal to noise ratio $\mathrm{SNR}=25 \mathrm{~dB}$.

$$
y_{k}=\sum_{i=1}^{3} C_{i}(k) \cdot \operatorname{Sin}\left(2 . \pi f_{i} \cdot T_{e} \cdot k+\beta_{i}(k)\right)+v_{k}
$$


Frequencies, amplitudes and phases of the three components are slowly time varying to prove the tracking capability of the estimation. The sampling rate is $F_{e}=2 \mathrm{~Hz}$ and the 3 frequencies are set initially to $f_{1}=0.2 \mathrm{~Hz}, f_{2}=0.3 \mathrm{~Hz}, f_{3}=0.4 \mathrm{~Hz}$ and vary as in Figure (13).

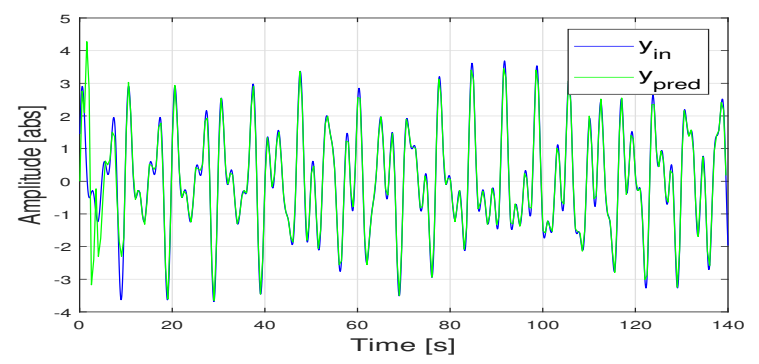

Fig. 1: Signal (blue) and its ANF prediction (green)

\section{A. Frequencies estimation by cascaded ANF and ARML}

The first stage estimates the three cascaded Notch Filter parameters. The parameters estimates $\hat{a}_{i}(0)$ are set to zero at the beginning. All ANF bandwidths are initially set at $r_{0}=0.1$ and exponentially increases to $r_{f}=0.8$ with a factor of $r_{d}=$ 0.99 (see equation 19). The figure 2 depicts the evolution of the ANF parameters estimation $\hat{a}_{i}(k)$ versus time $\left(t=k T_{e}\right)$. The dotted lines represent the true values of these parameters. The convergence is fast and estimations are precise and accurate. This results in a good (one step ahead) prediction of the signal as shown as in Figure (1).

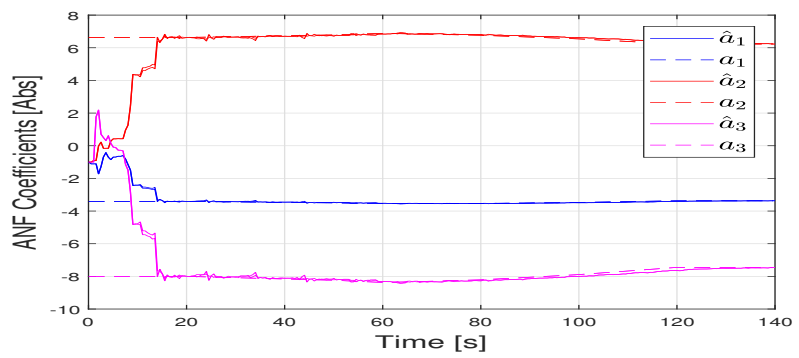

Fig. 2: Notch Filters parameters estimates versus time

The frequencies deduced, on line, from the estimated parameters using equations 7 . The following figure (3) illustrates the good convergence of the on line frequency estimations, after a short transient period (less than 20s). We observe that the frequency estimation converges after 36 sampling points (18s) and tracks simultaneous linear variations of the instantaneous frequencies.

\section{B. Amplitude and phase estimation by WRLS}

Using previous estimated frequencies, this second stage estimates amplitudes $C_{i}$ and phases $\beta_{i}$ of each one of the three components, using the proposed Weighted Recursive Least Squares algorithm. The regression vector and the parameters vector to be estimated are defined in the equation (4) and the setimations are given by equations (5 and II-A).

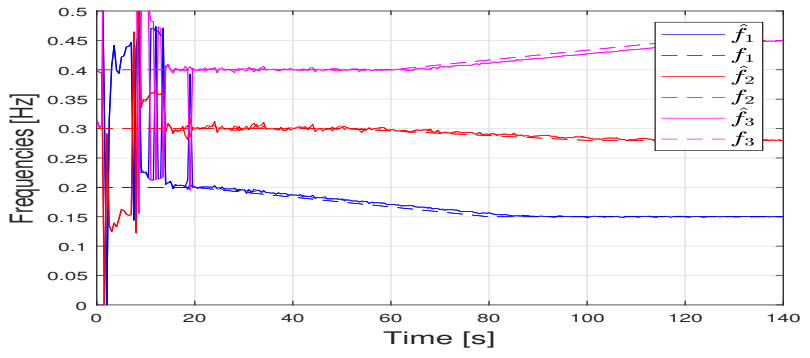

Fig. 3: Frequencies estimates versus time

The initial value of the adaptation gain is set to $G_{0}=100$ and the forgetting factor lambda $\lambda_{0}$ is set to 0.99 . The parameters vector $\hat{\theta}$ is initially set to 0 . Components of $\hat{\theta}: g_{l}$ and $h_{l}$ are used to compute $C_{i}$ and $\beta_{i}$ using the last equation of 5 .

The following figures illustrate the convergence of WRLS for amplitude/phase estimation. The figure 4 depicts the evolution of components amplitudes estimates versus time.

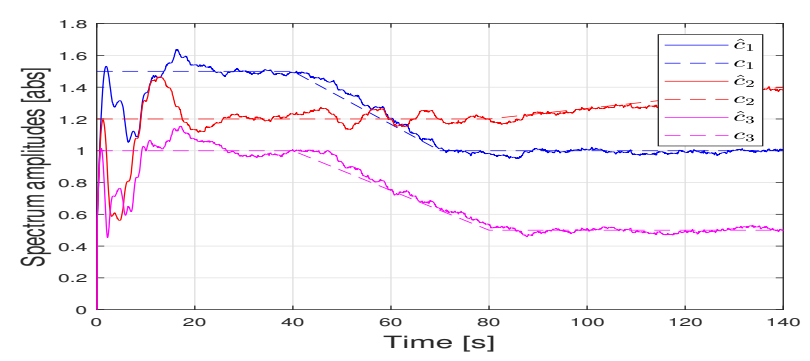

Fig. 4: Amplitudes estimates versus time

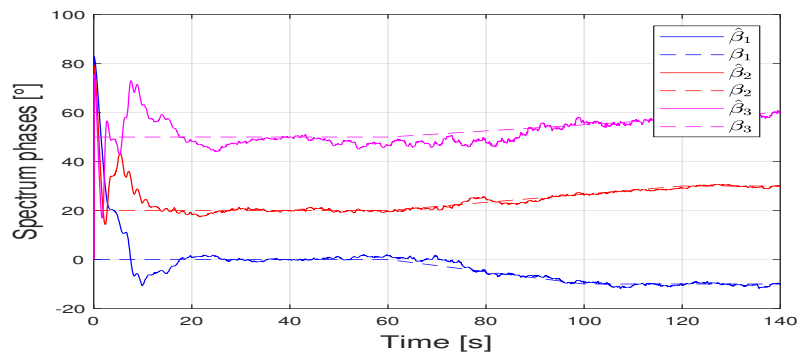

Fig. 5: Phases estimates versus time

The figure 4 shows the evolution of components phases estimates versus time. We observe that the amplitude/phase estimation converges after 50 points (25s) and tracks simultaneous linear variations of amplitudes and phases. The last figure shows that the prediction is quite good for a signal with 6 frequency components.

\section{CONClusion}

The main interest of the ANF in Cascade form is to simplify computation of the estimated frequencies and in case of independently time varying frequencies. A second order filter model appears to be faster to track variation than a higher order one.

The main results in this paper demonstrate the good performances observed in simulation for the ANF. This confirm the theoretical analysis and convergence proves (for RML and ELS PAAs). The exponential convergence of the estimation of the amplitudes and phases has been also proved. 

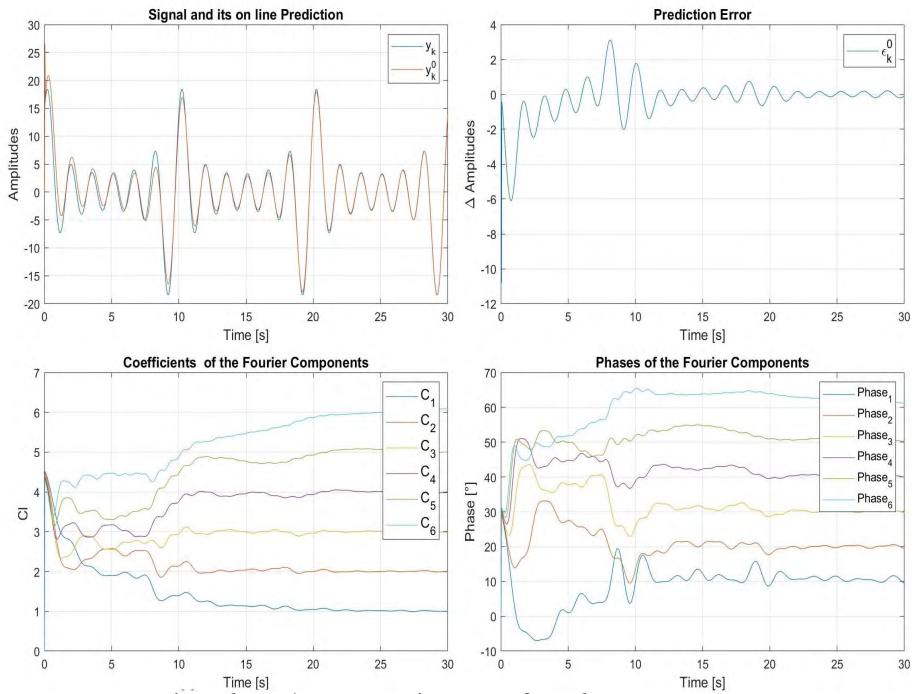

Fig. 6: Phases estimates for 6 components

The proposed (OPEM-RML) algorithm is asymptotically consistent and robust faced with the neglected dynamics and noise. In addition, for case of time varying signals, its tracking capabilities insure very good estimations and tracking of the parameters time variation.

Several estimation algorithms may be applied with these structures, like RELS, ARML, RIV (Recursive Extended Least Squares, Approximate Recursive Maximum Likelihood, Recursive Instrumental Variables with WRLS). Some special procedures may be employed to increase the convergence rate (by adjusting the bandwidth parameter $r$ ) and to enhance the estimation consistency and reduce the algorithm complexity.

The obtained estimation accuracy is better with the RML Adaptation Algorithm than with the Recursive Extended Least Squares method for which an upper limit of the de-biasing parameter is crucial in order to have a convergence without local instabilities.

The exponential convergence of the RIV-WRLS estimates of amplitudes and phases is proved by use of the theoretical background on persistent excitation. These results are very important for the time varying systems or in case of neglected dynamics due to the resulting robustness of the proposed algorithms. The implementation form studied here is computationally attractive and robust also when frequency, amplitude or phase are time varying.

\section{ACKNOWLEDGMENT}

The first author would like to thank I.D. Landau, for discussions and the suggestion to publish these results. He is also grateful for all the past collaborations they had. He addresses also many thanks to Odile Macchi for the collaborations. This work started in the $\mathrm{LaG}$ of ENSIEG in Grenoble, and is, now, founded by the SASV group of the LIS (UMR CNRS 7020).

\section{REFERENCES}

[1] I. D. Landau, M. Alma, A. Constantinescu, J. J. Martinez,

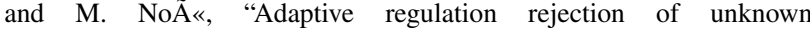
multiple narrow band disturbances (a review on algorithms and applications)," Control Engineering Practice, vol. 19, no. $10, \quad$ pp. $1168-1181,2011 . \quad$ [Online]. Available: http://www.sciencedirect.com/science/article/pii/S0967066111001213

[2] I. D. Landau, A. C. Silva, T.-B. Airimitoaie, G. Buche, and M. Noê̂š, "Benchmark on adaptive regulation rejection of unknown/time-varying multiple narrow band disturbances," European Journal of Control, vol. 19, no. 4, pp. 237 - 252, 2013, benchmark on Adaptive Regulation: Rejection of unknown/timevarying multiple narrow band disturbances. [Online]. Available: http://www.sciencedirect.com/science/article/pii/S0947358013000721

[3] P. Tomei, "Multi-sinusoidal disturbance rejection for discrete-time uncertain stable systems," Automatica, vol. 79, pp. $144-151,2017 . \quad$ [Online]. Available: http://www.sciencedirect.com/science/article/pii/S0005109817300481

[4] T.-B. Airimitoaie, "Robust Design and Tuning of Active Vibration Control Systems," Theses, Université de Grenoble, Jun. 2012. [Online]. Available: https://tel.archives-ouvertes.fr/tel-00767979

[5] B. Widrow, J. R. Glover, J. M. McCool, J. Kaunitz, C. S. Williams, R. H. Hearn, J. R. Zeidler, J. E. Dong, and R. C. Goodlin, "Adaptive noise cancelling: Principles and applications," Proceedings of the IEEE, vol. 63, no. 12, pp. 1692-1716, Dec 1975.

[6] I. Landau, M. M'Saad, N. M'Sirdi, and C. Canudas, "Identification of a Heat Exchanger Using an Apple II Computer." Revue generale de thermique, vol. 24, no. 280, 1985.

[7] I. D. Landau, N. K. M'Sirdi, and M. M'Saad, “Techniques de modÃ@lisation rÃ@cursives pour l'analyse spectrale param $\tilde{A} \odot$ trique adaptative," Traitement du Signal, vol. 3, no. 4-5, pp. 183 - 204, 1986. [Online]. Available: http://nkms.free.fr/RevueTS1986.pdf

[8] K. GLOVER, "Adaptive noise canceling applied to sinusoidal interferences," in IEEEASSP, IEEE International Conference on Acoustics, Speech and Signal Processing - Proceedings, vol. 25, no. 6, 1977.

[9] N. M'Sirdi, H. Tjokronegoro, and I. Landau, "RML ALGORITHM FOR RETRIEVAL OF SINUSOIDS WITH CASCADED NOTCH FILTERS." in ICASSP, IEEE International Conference on Acoustics, Speech and Signal Processing - Proceedings, 1988.

[10] D. B. RA0 and S. KUNG, "Adaptive notch filtering for retrieval of sinusoids in noise," IEEE Trans. ASSP, vol. 32, 1984. [Online]. Available: Available from: https://zapdf.com/adaptive-notch-filteringfor-the-retrieval-of-sinusoids-in-n.html

[11] A. Nehorai, "A minimal parameter adaptive notch filter with constrained poles and zeros," in ICASSP '85. IEEE International Conference on Acoustics, Speech, and Signal Processing, vol. 10, Apr 1985, pp. 11851188.

[12] N. M'Sirdi, O. Macchi, and J. Zarader, "Behaviour analysis of adaptive ARMA predictors with non-stationary inputs," in ICASSP, IEEE International Conference on Acoustics, Speech and Signal Processing Proceedings, vol. 3, 1990.

[13] N. K. M'Sirdi, H. R. Tjokronegoro, and I. D. Landau, "An rml algorithm for retrieval of sinusoids with cascaded notch filters," in ICASSP-88., International Conference on Acoustics, Speech, and Signal Processing, Apr 1988, pp. 2484-2487 vol.4.

[14] N. M'Sirdi and I. Landau, "ADAPTIVE EVOLUTIONARY SPECTRUM ANALYSIS FOR NARROW BAND SIGNALS." in ICASSP, IEEE International Conference on Acoustics, Speech and Signal Processing - Proceedings, 1987.

[15] N. K. M'Sirdi and J. L. Zarader, "Adaptive comb filters for enhancement of quasi periodic signals," in International Conference on Acoustics, Speech, and Signal Processing, Apr 1990, pp. 1461-1464 vol.3.

[16] I. D. Landau, "A feedback system approach to adaptive filtering," IEEE Trans. on Information Theory, March 1984.

[17] R. M. JOHNSON and col., "Exponential convergence of rls with exponential forgetting factor," System and control letters, vol. 2, no. 2, august 1982. 